\title{
ERGODIC SEQUENCES OF PROBABILITY MEASURES ON COMMUTATIVE HYPERGROUPS
}

\author{
LILIANA PAVEL
}

Received 5 May 2003

\begin{abstract}
We study conditions on a sequence of probability measures $\left\{\mu_{n}\right\}_{n}$ on a commutative hypergroup $K$, which ensure that, for any representation $\pi$ of $K$ on a Hilbert space $\mathscr{H}^{\pi}$ and for any $\xi \in \mathscr{H}^{\pi},\left(\int_{K} \pi_{X}(\xi) d \mu_{n}(x)\right)_{n}$ converges to a $\pi$-invariant member of $\mathscr{H}^{\pi}$.
\end{abstract}

2000 Mathematics Subject Classification: 43A65, 43A62, 43A30.

1. Introduction. The mean ergodic theorem was originally formulated by von Neumann [13] for one-parameter unitary groups in Hilbert space. Riesz [11] and Yosida [16] gave the first simple proofs that if $T$ is a bounded linear operator on a reflexive Banach space with subunitary norm, the corresponding sequence of averages $\left(A_{n}\right)_{n}$, $A_{n}=n^{-1} \sum_{k=0}^{n-1} T^{k}$, converges in the strong topology to a projection whose range is the set of fixed points of $T$. In a general operator semigroup, the elementary arithmetic means are not available. Eberlein [4] showed that the mean ergodic theorem depended in this setting on the existence of a net of averages with certain properties, calling the operator semigroup ergodic if such kind of a net can be found. Ergodic theorems in relation to the amenability concept were studied for the first time by Day. In [3], he proved that every bounded abelian operator semigroup is ergodic (in the Eberlein sense) as a consequence of the fact that every abelian semigroup is amenable. Greenleaf considered summing sequences of sets in amenable locally compact groups, relating them to ergodic theorems [6]. These are defined as sequences of compact sets $\left(U_{n}\right)_{n}$ covering $G$ having the properties that int $U_{n+1} \supseteq \operatorname{int} U_{n}$, for all $n$ and that the sequence $\left(\left|g U_{n} \Delta U_{n}\right| /\left|U_{n}\right|\right)_{n}$ converges to zero for each $g \in G$ (where $|E|$ is the left Haar measure of $E$ in $G$ ). Greenleaf showed that if $G \times S \rightarrow S$ is a measurable action of $G$ on a measure space $(S, \mathscr{B}, \mu)$ such that $\mu$ is $G$-invariant, then for $f \in L^{p},\left(\left|U_{n}\right|^{-1} \int_{U_{n}} f(g s) d g\right)_{n}$ converges in $L^{p}$ to a $G$-invariant function.

In [1], Blum and Eisenberg confined their attention only to the case of locally compact abelian groups (which are automatically amenable), but more general summing sequences for the purpose of ergodic theorems were found and shown to include ordinary Greenleaf summing sequences; representational techniques in connection to classical results in harmonic analysis on locally compact abelian groups came into play. They proved that if $G$ is a locally compact abelian group and $\left(\mu_{n}\right)_{n}$ is a sequence of probability measures on $G$, the following are equivalent:

(i) $\left(\mu_{n}\right)_{n}$ is strongly ergodic $\left(\left(\mu_{n}\right)_{n}\right.$ is a "generalized summing sequence" in [1]) meaning that, for every continuous unitary representation $\pi$ of $G$ on a Hilbert 
space $\mathscr{H}^{\pi}$ and for every $\xi$ in $\mathscr{H}^{\pi}$, the sequence $\left(\pi_{\mu_{n}}(\xi)\right)_{n}$ converges in norm to a $\pi$-invariant element of $\mathscr{H}^{\pi}$,

(ii) $\left(\hat{\mu}_{n}(\chi)\right)_{n}$ converges to zero for all $\chi \in \hat{G} \backslash\{1\}$,

(iii) $\left(\mu_{n}\right)_{n}$ converges weakly to the Haar measure on the Bohr compactification of $G$.

This result was generalized by Milnes and Paterson [10] to the case of second countable locally compact groups. They showed that for this class of locally compact groups, condition (i) is equivalent to the following:

(ii') $\left(\pi_{\mu_{n}}\right)_{n}$ converges to zero in the weak operator topology, for every irreducible continuous unitary representation $\pi$ of $G$ not equal to the identity representation.

$\left(\mathrm{iii}{ }^{\prime}\right)\left(\hat{\mu}_{n}\right)_{n}$ converges in the weak* topology to the unique invariant mean on $B_{I}(G)$, the closure in $C(G)$ of the linear span of the set of coefficient functions of the irreducible representations of $G$.

More recently, in [9], Lau and Losert obtained an improvement of this result, replacing $B_{I}(G)$ by the Fourier-Stieltjes algebra $B(G)$, for any $G$, and removing the condition of separability (second countability).

As it can be seen, the Blum-Eisenberg result has been extended to the general locally compact groups context, but as far as we know, it has not been studied in connection to hypergroups. The aim of the present paper is to generalize the Blum and Eisenberg mean ergodic theorem to the case of commutative hypergroups. A similar approach can be obtained for compact hypergroups.

Roughly speaking, hypergroups are locally compact spaces whose regular complex valued Borel measures form an algebra which has similar properties to the convolution algebra $(M(G), *)$ of a locally compact group $G$. Representations theory as well as harmonic analysis have been developed especially for commutative hypergroups and compact hypergroups. Unfortunately, even for the case of commutative hypergroups, one realises immediately that the space of almost periodic functions (investigated by Lasser [8] and Wolfenstetter [15]) is, in general, far from being an algebra. Likewise, the Bohr compactification does not carry any extending hypergroup structure, thus there is no hope to get an exact replacement of (iii) in Blum and Eisenberg theorem. On the other hand, in [12], Skantharajah studied amenability results on hypergroups, establishing in particular that the commutative (and the compact) hypergroups are amenable. Actually, as an immediate consequence of a result of Wolfenstetter [15], for commutative hypergroups, it can be shown that the invariant mean on $B(K)$, the closure in $C(K)$ of the linear span of the set of coefficient functions of the representations of $K$, is unique. These facts provide the necessary tools in order to obtain an appropriate variant of (iii) of Blum and Eisenberg theorem in similar terms to the ones of Milnes and Paterson approach [10].

2. Preliminary results. Throughout this paper, we will consider hypergroups in the sense of Jewett [7], a paper that may be consulted for basic definitions and results on hypergroups. The notation will be the same as here except for the following: $x \mapsto x^{\vee}$ denotes the involution on the hypergroup $K, \delta_{x}$ the Dirac measure concentrated at $x$, and $C(K)$ the bounded continuous complex valued functions on $K$. In addition, the set of probability measures on $K$ (with the cone topology, see [7, 2.2]) will be denoted by 
$M_{p}(K)$ and its subset consisting of measures with compact support (which with the convolution and the cone topology is a topological semigroup) by $P_{o o}(K)$. Following Jewett, [7, 11.3], we define a representation of $K$ as a norm increasing $*$-representation of the Banach $*$-algebra $M(K)$. Note that if $\pi$ is a representation of $K$ in some Hilbert space $\mathscr{H}^{\pi}$ and $\mu \in M(K), \pi_{\mu}$ is a bounded linear operator on $\mathscr{H}^{\pi}$ with $\left\|\pi_{\mu}\right\| \leq\|\mu\|$ (so $\pi_{\mu}$ is an element of the space of bounded linear operators on $\mathscr{H}^{\pi}, \mathscr{B}\left(\mathscr{H}^{\pi}\right)$ ). For notational convenience, we write $\pi_{x}$ for $\pi_{\delta_{x}}$, where $x \in K$.

The space (which is not an algebra) of weakly almost periodic functions on a hypergroup $K$, WAP $(K)$, has been studied by Wolfenstetter [15]. We enlight the connection with hypergroups representations.

Proposition 2.1. Let $\pi$ be a representation of a hypergroup $K$ on some Hilbert space $\left(\mathscr{H}^{\pi},\langle\cdot, \cdot\rangle\right)$. Then, for every $\xi, \eta \in \mathscr{H}^{\pi}$, the mapping on $K, x \mapsto F_{\xi, \eta}(x)$,

$$
F_{\xi, \eta}(x)=\left\langle\pi_{x}(\xi), \eta\right\rangle, \quad \forall x \in K
$$

belongs to $\mathrm{WAP}(K)$.

Proof. The proof closely follows the one for locally compact groups case. Indeed, embed $K$ into the topological semigroup $P_{o o}(K)$, by the homeomorphism $x \mapsto \delta_{x}$ (see [7, Lemma 2.2B]). Then $\mu \mapsto \pi_{\mu}$ is a representation of $P_{o o}(K)$ in $\mathscr{B}\left(\mathscr{H}^{\pi}\right)$ and $\left\|\pi_{\mu}\right\| \leq 1$, for all $\mu \in P_{o o}(K)$. Since $\left\{\pi_{\mu}(\xi) \mid \mu \in P_{o o}(K)\right\}$ is bounded in the Hilbert space $\mathscr{H}^{\pi}$, it results that it is a relatively weak compact. As the function $F_{\xi, \eta}$ can be extended to $P_{o o}(K)$ by $F_{\xi, \eta}(\mu)=\Phi\left(\pi_{\mu}(\xi)\right)$, where $\Phi \in\left(\mathscr{H}^{\pi}\right)^{*}, \Phi(\zeta)=\langle\zeta, \eta\rangle$, using [2, Theorem 3.1(B)], we can infer that the function $F_{\xi, \eta}$ is weakly almost periodic on $P_{o o}(K)$, consequently on $K . \square$

Notation. Denote by $B(K)$ the closure in $C(K)$ of the linear span of the set of coefficient functions of the representations of $K$. Such a coefficient function is of the form $F_{\xi, \eta}(x)=\left\langle\pi_{x}(\xi), \eta\right\rangle$, for $\pi$ is a representation of $K$ on a Hilbert space $\mathscr{H}^{\pi}$ and $\xi$, $\eta \in \mathscr{H}^{\pi}$.

COROLLARY 2.2. The closure $B(K)$ is contained in $\mathrm{WAP}(K)$.

Proof. The proof is clear using the fact that $\mathrm{WAP}(K)$ is a norm closed linear subspace of $C(K)$ (see [15, Proposition 2.3]).

Corollary 2.3. Any bounded positive definite function on $K$ belongs to $\operatorname{WAP}(K)$.

Proof. The proof is a consequence of the previous proposition and of $[7$, Theorem 11.4B].

Wolfenstetter proved that every commutative hypergroup $K$ posseses a unique left invariant mean on $\mathrm{WAP}(K)$ (see [15, Theorem 2.11]). We recall that a left invariant mean is a continuous positive linear functional $m$ on $\mathrm{WAP}(K)$ with the operatorial norm $\|m\|=1$ such that (i) $m(f)=m\left(L_{x} f\right)$, for all $f \in \mathrm{WAP}(K), x \in K$; (ii) $m(1)=1$; (iii) $m(\bar{f})=\overline{m(f)}$, for all $f \in \operatorname{WAP}(K)$. Using the cited theorem and taking into account that $1 \in B(K)$, we have, restricting the left invariant mean of $\mathrm{WAP}(K)$ to $B(K)$, the next result. 
Proposition 2.4. For any commutative hypergroup $K$, there is a unique left invariant mean on $B(K)$.

Further, we recall the notion of ergodic sequences ("generalized summing sequence" in [1]) of probability measures $[1,10]$.

If $\pi$ is a representation of a hypergroup $K$ on some Hilbert space $\mathscr{H}^{\pi}$, the fixed-point set of $\pi$ is

$$
\mathscr{H}_{f}^{\pi}=\left\{\xi \in \mathscr{H}^{\pi} \mid \pi_{x}(\xi)=\xi, \forall x \in K\right\}
$$

We notice that $\mathscr{H}_{f}^{\pi}$ is a closed linear subspace of $\mathscr{H}^{\pi}$; in addition,

$$
\mathscr{H}_{f}^{\pi}=\left\{\xi \in \mathscr{H}^{\pi} \mid \pi_{\mu}(\xi)=\xi, \forall \mu \in M_{p}(K)\right\} .
$$

DEFINITION 2.5. A sequence $\left(\mu_{n}\right)_{n}$ in $M_{p}(K)$ is called strongly (resp., weakly) ergodic if, for every representation $\pi$ of $K$ on a Hilbert space $\mathscr{H}^{\pi}$ and for every $\xi \in \mathscr{H}^{\pi}$, the sequence $\left(\pi_{\mu_{n}} \xi\right)_{n}$ converges in norm (resp., weakly) to a member of $\mathscr{H}_{f}^{\pi}$.

REMARK 2.6. The key of the study of ergodic sequences on locally compact groups is the fact that the above definition can be given in terms of strong (resp., weak) convergence of the operators sequence $\left(\pi_{\mu_{n}}\right)_{n}$ in $\mathscr{B}\left(\mathscr{H}^{\pi}\right)$. Despite the fact that the operators of a hypergroup representation are not unitary, we have a similar situation in the case of hypergroups. More precisely, we have the following proposition.

Proposition 2.7. A sequence of probability measures $\left(\mu_{n}\right)_{n}$ is strongly (resp., weakly) ergodic if and only if for every representation $\pi$ of $K$ on a Hilbert space $\mathcal{H}^{\pi}$, the sequence $\left(\pi_{\mu_{n}}\right)_{n}$ converges in the strong operator topology (resp., in the weak operator topology) to $P^{\pi}$, the orthogonal projection onto $\mathcal{H}_{f}^{\pi}$.

PRoof. The proof for locally compact groups case can be easily applied to hypergroups with only minor changes, using the norm-increasing property of the hypergroups representations operators. We sketch this proof here (adapting it to our approach from Godement [5]) for the sake of completeness. For $\pi$, a representation of $K$ on a Hilbert space $\mathscr{H}^{\pi}$ considers the operators semigroup $\mathscr{G}^{\pi}=\left\{\pi_{\mu} \mid \mu \in M_{p}(K)\right\}$. Clearly, each member of $\mathscr{G}^{\pi}$ has the norm subunitary. As $\mathscr{H}^{\pi}$ is uniformely convex, for each $\theta \in \mathscr{H}^{\pi}$, there exists a unique $a_{\theta}$ in

$$
A_{\theta}=\overline{\operatorname{co}\left\{\pi_{\mu}(\theta) \mid \mu \in M_{p}(K)\right\}}
$$

such that

$$
\left\|a_{\theta}\right\|=\inf _{b \in A_{\theta}}\|b\|
$$

For $\mu \in M_{p}(K)$ and $b \in A_{\theta}, b=\sum_{j=1}^{n} t_{j} \pi_{\mu_{j}}(\theta)$, it follows that $\pi_{\mu}(b)$,

$$
\pi_{\mu}(b)=\sum_{j=1}^{n} t_{j} \pi_{\mu * \mu_{j}}(\theta),
$$


is still in $A_{\theta}$, so we can infer that $\pi_{\mu}\left(a_{\theta}\right) \in A_{\theta}$. In addition,

$$
\left\|\pi_{\mu}\left(a_{\theta}\right)\right\| \leq\left\|\pi_{\mu}\right\| \cdot\left\|a_{\theta}\right\| \leq\left\|a_{\theta}\right\|
$$

which, taking into account that $\left\|a_{\theta}\right\|=\inf _{b \in A_{\theta}}\|b\|$, shows that $\left\|\pi_{\mu}\left(a_{\theta}\right)\right\|=\left\|a_{\theta}\right\|$. By the uniqueness of $a_{\theta}$ (realising the infimum of $\|b\|$ on $A_{\theta}$ ), it results that $\pi_{\mu}\left(a_{\theta}\right)=a_{\theta}$, for all $\mu \in M_{p}(K)$, hence $a_{\theta} \in \mathscr{H}_{f}^{\pi} \cap A_{\theta}$. Let $\theta^{\prime}=P^{\pi}(\theta)$, so $\theta=\theta^{\prime}+\alpha$, for some $\alpha$ in the orthogonal complement of $\mathscr{H}_{f}^{\pi}$. Then, $A_{\alpha} \subset\left(\mathscr{H}_{f}^{\pi}\right)^{\perp}$; therefore, the element which realises $\inf _{b \in A_{\alpha}}\|b\|$ is in $\mathscr{H}_{f}^{\pi} \cap\left(\mathscr{H}_{f}^{\pi}\right)^{\perp}=\{0\}$. It follows that, for each $\varepsilon>0$, one can find $k \in A_{\alpha}, k=\sum_{j=1}^{n} t_{j} \pi_{\mu_{j}}(\alpha)$, with $\|k\| \leq \varepsilon$. Thus we have

$$
\|k\|=\left\|\sum_{j=1}^{n} t_{j} \pi_{\mu_{j}}(\alpha)\right\|=\left\|\sum_{j=1}^{n} t_{j} \pi_{\mu_{j}}\left(\theta-\theta^{\prime}\right)\right\|=\left\|\sum_{j=1}^{n} t_{j} \pi_{\mu_{j}}(\theta)-\theta^{\prime}\right\|<\varepsilon,
$$

from where we infer that $\theta^{\prime} \in A_{\theta}$. On the other hand,

$$
\left\|\sum_{j=1}^{n} t_{j} \pi_{\mu_{j}}(\theta)\right\|^{2}=\left\|\sum_{j=1}^{n} t_{j} \pi_{\mu_{j}}(\alpha)+\theta^{\prime}\right\|^{2}=\left\|\sum_{j=1}^{n} t_{j} \pi_{\mu_{j}}(\alpha)\right\|^{2}+\left\|\theta^{\prime}\right\|^{2} \geq\left\|\theta^{\prime}\right\|^{2},
$$

so $\theta^{\prime}$ is the element of norm minim in $A_{\theta}$, that means $\theta^{\prime}=a_{\theta}$.

Now, for $\xi \in \mathscr{H}_{f}^{\pi}$, if $\left(\pi_{\mu_{n}}(\xi)\right)_{n}$ converges to a member of $\mathscr{H}_{f}^{\pi}$, it follows that this element is in $\mathscr{H}_{f}^{\pi} \cap A_{\xi}$, so it coincides with $P^{\pi}(\xi)$ which ends the proof.

Similar arguments can be done with respect to the weak ergodicity related to the weak convergence.

3. Main results. For a measure $\mu \in M(K)$, the function $\hat{\mu}$ on $C(K)$ (or any subspace of $C(K))$ is defined by

$$
\hat{\mu}(f)=\int f d \mu
$$

The next result generalizes the Blum-Eisenberg's mean ergodic theorem [1] from the commutative locally compact groups to the case of commutative hypergroups.

THEOREM 3.1. Let $K$ be a commutative hypergroup and $\left(\mu_{n}\right)_{n}$ a sequence of probability measures on $K$. The following are equivalent:

(i) $\left(\mu_{n}\right)_{n}$ is strongly ergodic;

(ii) for every character $x$ on $K$, not equal to the identity, the Fourier transforms sequence $\left(\hat{\mu}_{n}(\chi)\right)_{n}$ converges to 0 ;

(iii) the sequence $\left(\hat{\mu}_{n}\right)_{n}$ converges in the weak* topology to $m$, the unique invariant mean on $B(K)$.

Proof. (i) $\Rightarrow\left(\right.$ ii). Suppose that the sequence $\left(\hat{\mu}_{n}(\chi)\right)_{n}$ does not converge to zero for some $\chi \neq 1$. Consider the representation $\pi: M(K) \rightarrow B\left(\mathscr{H}^{\pi}\right), \pi_{\chi}(\xi)=\chi(\chi) \xi$, for all $\xi \in \mathscr{H}^{\pi}, x \in K$. As $\chi(x) \neq 1, \pi_{x}(\xi) \neq \xi$, for some $x \in K$, so $\mathscr{H}_{f}^{\pi}=\{0\}$. It follows that $P^{\pi}$, the orthogonal projection onto $\mathscr{H}_{f}^{\pi}$, is 0 . We show that $\left(\pi_{\mu_{n}}\right)_{n}$ does not converge 
pointwise to $P^{\pi}=0$. Indeed,

$$
\begin{aligned}
\left\|\int \pi_{x}(\xi) d \mu_{n}(x)\right\|^{2} & =\left\langle\int \pi_{x}(\xi) d \mu_{n}(x), \int \pi_{y}(\xi) d \mu_{n}(y)\right\rangle \\
& =\iint\left\langle\pi_{x}(\xi), \pi_{y}(\xi)\right\rangle d \mu_{n}(x) d \mu_{n}(y) \\
& =\iint \chi(x) \chi\left(y^{\vee}\right)\|\xi\|^{2} d \mu_{n}(x) d \mu_{n}(y) \\
& =\|\xi\|^{2} \iint \chi(x) \overline{\chi(y)} d \mu_{n}(x) d \mu_{n}(y) \\
& =\|\xi\|^{2}\left|\hat{\mu}_{n}(x)\right|^{2}
\end{aligned}
$$

that does not converge to zero.

(ii) $\Rightarrow$ (i). Assume that $\hat{\mu}_{n}(\chi) \rightarrow 0$, for all $\chi$ character not equal to the identity. Let $\pi$ be a representation of $K$ on some Hilbert space $\mathscr{H}^{\pi}$ and $P^{\pi}(\xi)$ the projection of $\xi \in \mathscr{H}^{\pi}$ onto $\mathcal{H}_{f}^{\pi}$. Denoting $\eta=\xi-P^{\pi}(\xi)$, as $\pi_{\mu_{n}} P^{\pi}(\xi)=P^{\pi}(\xi)$, we have

$$
\begin{aligned}
\left\|\pi_{\mu_{n}}(\xi)-P^{\pi}(\xi)\right\|^{2} & =\left\|\pi_{\mu_{n}}\left(\xi-P^{\pi}(\xi)\right)\right\|^{2} \\
& =\left\|\pi_{\mu_{n}}(\eta)\right\|^{2}=\left\|\int \pi_{x}(\eta) d \mu_{n}(x)\right\|^{2} \\
& =\left\langle\int \pi_{x}(\eta) d \mu_{n}(x), \int \pi_{y}(\eta) d \mu_{n}(y)\right\rangle \\
& =\iint\left\langle\pi_{x}(\eta), \pi_{y}(\eta)\right\rangle d \mu_{n}(x) d \mu_{n}(y) \\
& =\iint\left\langle\pi_{y^{\vee} * x}(\eta), \eta\right\rangle d \mu_{n}(x) d \mu_{n}(y) \\
& =\iint F_{\eta, \eta}^{\pi}\left(y^{\vee} * x\right) d \mu_{n}(x) d \mu_{n}(y) \\
& =\int F_{\eta, \eta}^{\pi}(z) d\left(\mu_{n} * \mu_{n}^{*}\right)(z) .
\end{aligned}
$$

As $F_{\eta, \eta}^{\pi}$ is a bounded positive definite function by the Bochner's theorem (see [7, Theorem 12.3B]), there exists a unique $a \in M^{+}(\hat{K})$ such that

$$
F_{\eta, \eta}^{\pi}(x)=\int_{\hat{K}} \chi(x) \mid d a(x), \quad \forall x \in K .
$$

It follows that

$$
\begin{aligned}
\int F_{\eta, \eta}^{\pi}(z) d\left(\mu_{n} * \mu_{n}^{*}\right)(z) & =\iint_{\hat{K}} \chi(z) d a(\chi) d\left(\mu_{n} * \mu_{n}^{*}\right)(z) \\
& =\int_{\hat{K}}\left(\int \chi(z) d\left(\mu_{n} * \mu_{n}^{*}\right)(z)\right) d \bar{a}(\chi) \\
& =\int_{\hat{K}}\left|\hat{\mu}_{n}(\chi)\right| d \bar{a}(\chi) .
\end{aligned}
$$

Consequently,

$$
\left\|\pi_{\mu_{n}}(\xi)-P^{\pi}(\xi)\right\|^{2}=\int_{\hat{K}}\left|\hat{\mu}_{n}(\chi)\right| d \bar{a}(\chi)=\int_{\hat{K} \backslash\{1\}}\left|\hat{\mu}_{n}(\chi)\right| d \bar{a}(\chi)+\bar{a}(\{\chi=1\}) .
$$


By Lebesgue dominated convergence theorem and assumption (i), this converges to $\bar{a}(\{X=1\})$. As the representation $\pi$ acts on $\mathcal{H}_{f}^{\pi}$ as the identity representation and $\eta$ is in the orthogonal complement of $\mathscr{H}_{f}^{\pi}$, it follows that $\bar{a}(\{X=1\})=0$. Otherwise, if $\bar{a}(\{\chi=1\})>0$, the identity representation would have to be a subrepresentation of the subrepresentation of $\pi$ acting on $\left(\mathscr{H}_{f}^{\pi}\right)^{\perp}$, which contradicts $\left.\pi\right|_{\mathscr{H}} ^{\pi}=I$.

(i) $\Rightarrow$ (iii). First we notice that, for $F_{\xi, \eta}^{\pi} \in B(K)$,

$$
\hat{\mu}_{n}\left(F_{\xi, \eta}^{\pi}\right)=\int F_{\xi, \eta}^{\pi}(x) d \mu_{n}(x)=\int\left\langle\pi_{x}(\xi), \eta\right\rangle d \mu_{n}(x)=\left\langle\pi_{\mu_{n}}(\xi), \eta\right\rangle
$$

so the sequence $\left(\hat{\mu}_{n}\left(F_{\xi, \eta}^{\pi}\right)\right)_{n}$ converges to $\left\langle P^{\pi}(\xi), \eta\right\rangle$. On the other hand, as

$$
L_{y} F_{\xi, \eta}^{\pi}(x)=F_{\xi, \eta}^{\pi}(y * x)=\left\langle\pi_{y * x}(\xi), \eta\right\rangle=\left\langle\pi_{x}(\xi), \pi_{y \vee}(\eta)\right\rangle=F_{\xi, \pi_{y \vee}(\eta)}^{\pi}(x)
$$

we can infer that $\left(\hat{\mu}_{n}\left(L_{y} F_{\xi, \eta}^{\pi}\right)\right)_{n}$ converges to $\left\langle P^{\pi}(\xi), \pi_{y} \vee(\eta)\right\rangle$. Since

$$
\left\langle P^{\pi}(\xi), \pi_{y} \vee(\eta)\right\rangle=\left\langle\pi_{y}\left(P^{\pi}(\xi)\right), \eta\right\rangle=\left\langle P^{\pi}(\xi), \eta\right\rangle,
$$

it follows that both sequences $\left(\hat{\mu}_{n}\left(L_{y} F_{\xi, \eta}^{\pi}\right)\right)_{n}$ as well as $\left(\hat{\mu}_{n}\left(F_{\xi, \eta}^{\pi}\right)\right)_{n}$ converge to $\left\langle P^{\pi}(\xi), \eta\right\rangle$.

Therefore, $\left(\hat{\mu}_{n}\right)_{n}$ converges in the weak* toplogy to $m$, the unique left invariant mean on $B(K)$.

(iii) $\Rightarrow$ (ii). Let $\chi \in \hat{K} \backslash\{1\}$ and $\pi: M(K) \rightarrow B\left(\mathscr{H}^{\pi}\right)$, the representation defined by $\pi_{x}(\xi)=$ $\chi(x) \xi$, for all $\xi \in \mathscr{H}^{\pi}, x \in K$. As

$$
\left\langle\pi_{\mu_{n}}(\xi), \eta\right\rangle=\hat{\mu}_{n}\left(F_{\xi, \eta}^{\pi}\right) \longrightarrow m\left(F_{\xi, \eta}^{\pi}\right), \quad \forall \xi, \eta \in \mathscr{H}^{\pi},
$$

it follows that $\left(\pi_{\mu_{n}}\right)_{n}$ converges to some $T \in \mathscr{B}\left(\mathscr{H}^{\pi}\right)$ in the weak operator topology. Since $\langle T(\xi), \eta\rangle=m\left(F_{\xi, \eta}^{\pi}\right)$, by the left invariance of $m$, we have $\pi_{x} T=T=T \pi_{x}$, for all $x \in K$, that means

$$
\pi_{x}(T)(\xi)=\chi(x) T(\xi)=T(\xi), \quad \forall \xi \in \mathscr{H}^{\pi} .
$$

As $\chi(x) \neq 1$, it follows that $T(\xi)=0$, for all $\xi \in \mathscr{H}^{\pi}$. For $\xi \in \mathscr{H}^{\pi}$ arbitrary, we have

$$
\begin{aligned}
0 & =\langle T(\xi), \xi\rangle=\lim _{n} \int\left\langle\pi_{x}(\xi), \xi\right\rangle d \mu_{n}(x) \\
& =\lim _{n}\|\xi\|\left(\int \chi(x) d \mu_{n}(x)\right)=\left(\lim _{n} \hat{\mu}_{n}(x)\right)\|\xi\|^{2},
\end{aligned}
$$

so $\hat{\mu}_{n}(\chi) \rightarrow 0$, for all $\chi \neq 1$.

Proposition 3.2. Let $K$ be a commutative hypergroup and $\left(\mu_{n}\right)_{n}$ a weakly ergodic sequence of probabilities on $K$. Then $\left(\mu_{n}\right)_{n}$ is strongly ergodic. 
Proof. Let $\left(\mu_{n}\right)_{n}$ be a weakly ergodic sequence of probabilities. Whenever $\pi$ is an irreducible representation of $K,\left(\pi_{\mu_{n}}\right)_{n}$ converges strongly to $P^{\pi}$ because $\pi$ is finitedimensional (actually, it is one-dimensional). Moreover, if $\pi$ does not coincide with the identity representation, $P^{\pi}=0$. From here, we get with similar arguments as in the proof of Theorem $3.1((\mathrm{ii}) \Rightarrow(\mathrm{i}))$ that for each arbitrary representation $\pi$ of $K$,

$$
\left\|\pi_{\mu_{n}} \xi-P^{\pi}(\xi)\right\| \longrightarrow 0
$$

Thus $\left(\pi_{\mu_{n}} \xi\right)_{n}$ converges strongly to $P^{\pi}$.

REMARK 3.3. We notice that as any representation of a compact hypergroup is finitedimensional [14], similar results to the studied case of commutative hypergroups can be obtained for compact hypergroups. In this situation, the unique invariant mean on $B(K)$ is the restriction of the Haar measure of $K$.

EXAMPLE 3.4. Let $K$ be a commutative hypergroup and $\lambda$ its left Haar measure (it is known that the commutative hypergroups possess Haar measure). If $\left(U_{n}\right)_{n}$ is a sequence of sets of $K$ such that

$$
\lim _{n} \frac{\lambda\left(U_{n} \cap x * U_{n}\right)}{\lambda\left(U_{n}\right)}=1, \quad \forall x \in K
$$

then the sequence $\left(\mu_{n}\right)_{n}$, defined by $\mu_{n}(A)=\lambda\left(A \cap U_{n}\right) / \lambda\left(U_{n}\right)$, is strongly ergodic. This follows from Theorem 3.1, (ii) $\Rightarrow(\mathrm{i})$. Indeed, take $\chi \in \hat{K} \backslash\{1\}$. Then, there exists $x_{o} \in K$ such that $\chi\left(x_{o}\right) \neq 1$. As

$$
\frac{\chi\left(x_{o}\right)}{\lambda\left(U_{n}\right)} \int_{U_{n}} \chi(x) d \lambda(x)=\frac{1}{\lambda\left(U_{n}\right)} \int_{x_{o} * U_{n}} \chi(x) d \lambda(x)
$$

it results that $\left(\int_{U_{n}} \chi(x) d \lambda(x) / \lambda\left(U_{n}\right)\right)_{n}$ converges to 0 , so equivalently, $\left(\hat{\mu}_{n}(\chi)\right)_{n}$ converges to 0 .

\section{REFERENCES}

[1] J. Blum and B. Eisenberg, Generalized summing sequences and the mean ergodic theorem, Proc. Amer. Math. Soc. 42 (1974), 423-429.

[2] R. B. Burckel, Weakly Almost Periodic Functions on Semigroups, Gordon and Breach Science Publishers, New York, 1970.

[3] M. M. Day, Ergodic theorems for Abelian semigroups, Trans. Amer. Math. Soc. 51 (1942), 399-412

[4] W. F. Eberlein, Abstract ergodic theorems and weak almost periodic functions, Trans. Amer. Math. Soc. 67 (1949), 217-240.

[5] R. Godement, Les fonctions de type positif et la théorie des groupes, Trans. Amer. Math. Soc. 63 (1948), 1-84 (French).

[6] F. P. Greenleaf, Ergodic theorems and the construction of summing sequences in amenable locally compact groups, Comm. Pure Appl. Math. 26 (1973), 29-46.

[7] R. I. Jewett, Spaces with an abstract convolution of measures, Advances in Math. 18 (1975), no. 1, 1-101.

[8] R. Lasser, Almost periodic functions on hypergroups, Math. Ann. 252 (1980), no. 3, 183196. 
[9] A. T. Lau and V. Losert, Ergodic sequences in the Fourier-Stieltjes algebra and measure algebra of a locally compact group, Trans. Amer. Math. Soc. 351 (1999), no. 1, 417428.

[10] P. Milnes and A. L. T. Paterson, Ergodic sequences and a subspace of B $(G)$, Rocky Mountain J. Math. 18 (1988), no. 3, 681-694.

[11] F. Riesz, Some mean ergodic theorems, J. London Math. Soc. 13 (1938), 274-278.

[12] M. Skantharajah, Amenable hypergroups, Illinois J. Math. 36 (1992), no. 1, 15-46.

[13] J. von Neumann, Proof of the quasi-ergodic hypothesis, Proc. Natl. Acad. Sci. USA 18 (1932), 70-82.

[14] R. C. Vrem, Harmonic analysis on compact hypergroups, Pacific J. Math. 85 (1979), no. 1, 239-251.

[15] S. Wolfenstetter, Weakly almost periodic functions on hypergroups, Monatsh. Math. 96 (1983), no. 1, 67-79.

[16] K. Yosida, Mean ergodic theorem in Banach spaces, Proc. Imp. Acad. Tokyo 14 (1938), 292-294.

Liliana Pavel: Faculty of Mathematics and Computer Science, Bucharest University, Academiei 14, 70109 Bucharest, Romania

E-mail address: 1pave1@1an.unibuc.ro 


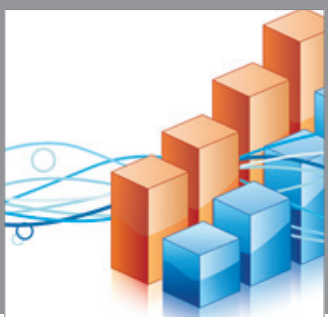

Advances in

Operations Research

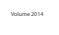

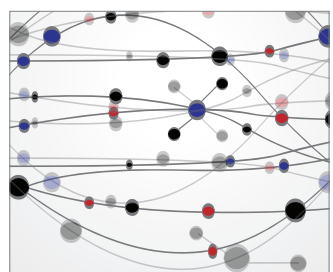

\section{The Scientific} World Journal
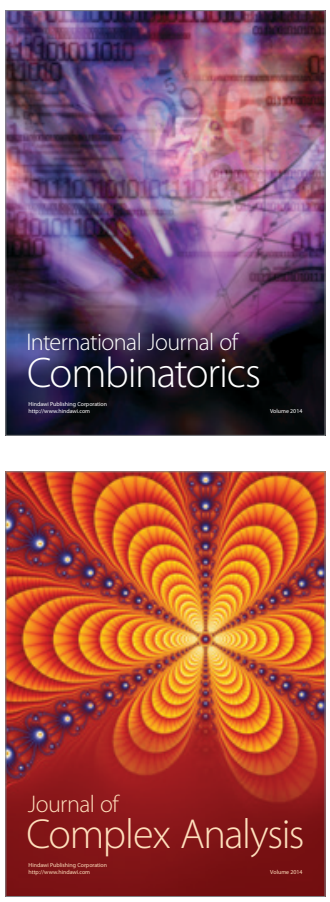

International Journal of

Mathematics and

Mathematical

Sciences
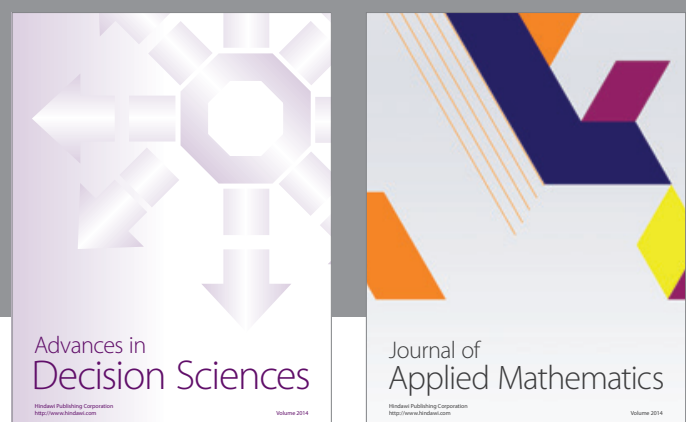

Journal of

Applied Mathematics
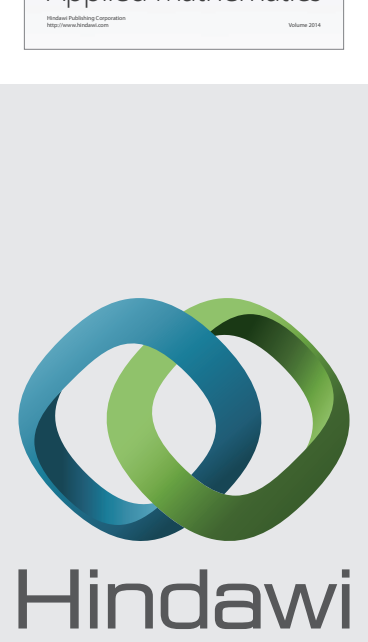

Submit your manuscripts at http://www.hindawi.com
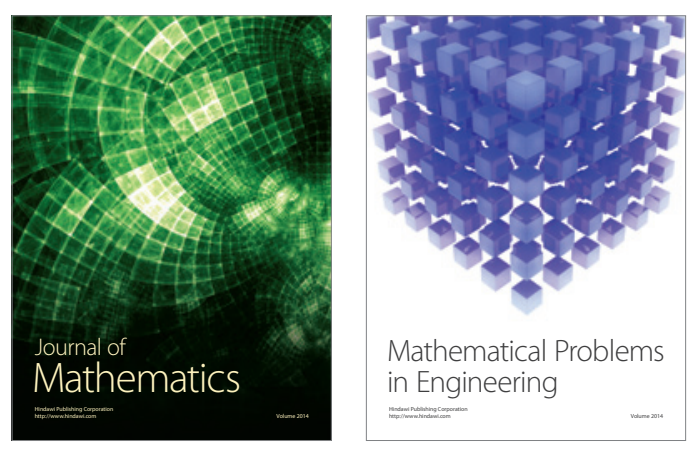

Mathematical Problems in Engineering
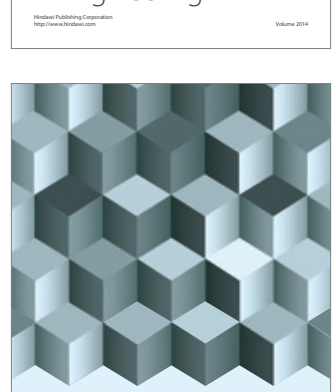

Journal of

Function Spaces
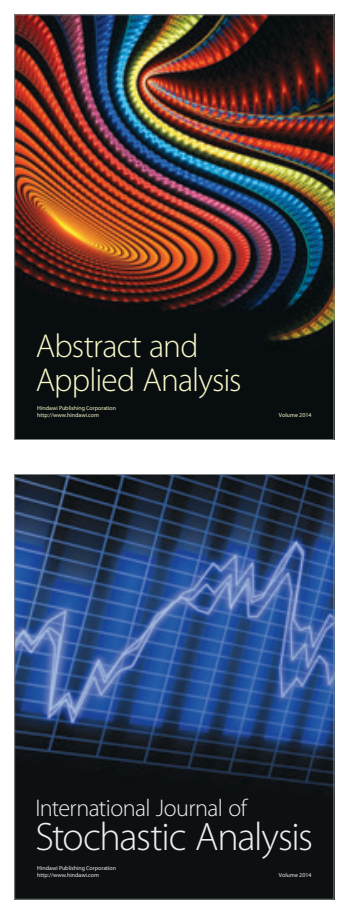

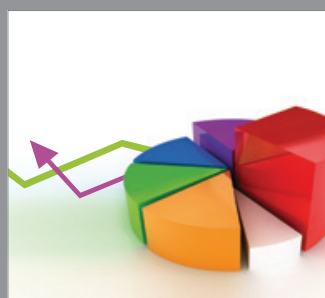

ournal of

Probability and Statistics

Promensencen
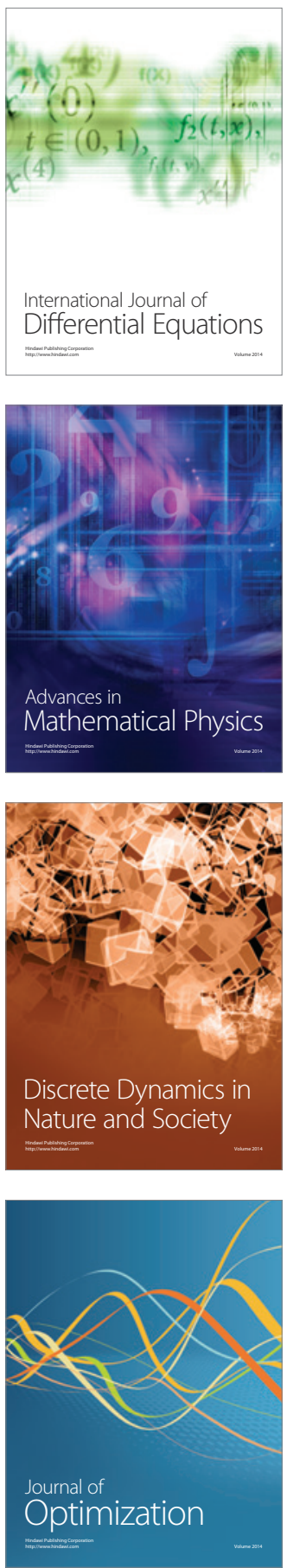